\title{
USO DE SÉRIES TEMPORAIS NA ANÁLISE DE VAZÃO DE ÁGUA NA REPRESA DE FURNAS
}

\author{
Use of time series analysis for the water outflow at furnas dam
}

\author{
Thelma Sáfadi ${ }^{1}$
}

\begin{abstract}
RESUMO
Com este trabalho teve-se como principal objetivo analisar o comportamento da série de vazão de água na barragem de Furnas, empregando análise de séries temporais e estudando o efeito de sazonalidade, tendência e intervenção. Para a análise, foram considerados modelos de séries temporais com e sem a presença de intervenção. Os dados referem-se à vazão $\left(\mathrm{m}^{3} / \mathrm{s}\right)$ da Barragem de Furnas - MG, coletada diariamente no período de janeiro de 1963 a dezembro de 1994. Foi realizada uma média mensal dos dados, em que cada média representa uma observação, num total de 372. Observou-se que a série de vazão fica bem ajustada utilizando modelos sazonais e a incorporação do parâmetro de intervenção forneceu informações complementares na análise.
\end{abstract}

Termos para indexação: Análise de intervenção, modelo SARIMA, vazão de água.

\section{ABSTRACT}

This work aimed to fit time series models to the series of water outflow at Furnas Dam. We studied the effect of trend, seasonality and intervention factor in the analysis. Data were colleted daily from January of 1963 to December of 1994 at Furnas Dam. We considered Box \& Jenkins models models with and without the intervention factor. We noted that the series of water outflow are fitted by SARIMA models and the intervention factor gave us more information.

Index Terms: Intervention analysis, SARIMA model, water flow.

(Recebido para publicação em 11 de setembro de 2002 e aprovado em 10 de fevereiro de 2003)

\section{INTRODUÇÃO}

As técnicas de análise de séries temporais vêm sendo utilizadas com freqüência em várias áreas de pesquisa, como: ciências sociais e políticas, economia, sociologia, história, psicologia, meio ambiente, entre outras, nas quais as observações estão geralmente associadas ao tempo e seus métodos evidenciam características dinâmicas dos fenômenos.

Existem vários métodos possíveis para ajustar um modelo para uma série temporal; neste trabalho, serão citados os modelos de Box e Jenkins e sua estimação será efetuada de forma iterativa pelo Método da Máxima Verossimilhança, em que o modelo geral é um ARIMA (Autorregressivo-IntegradoMédias Móveis ).

Na utilização dos modelos de Box e Jenkins, é necessário que a série seja estacionária, ou seja, não apresente tendência e sazonalidade. Para verificar a presença desses fatores, é necessário aplicar alguns testes apropriados. $\mathrm{O}$ teste proposto para analisar o efeito da tendência é o de Cox-Stuart, conhecido como teste da tendência ou do sinal (MORETTIN e TOLOI, 1985) e para verificar a existência de sazonalidade na série, considerou-se a função de autocorrelação e uma análise do periodograma.

Para um bom ajuste do modelo de Box e Jenkins, é necessário utilizar técnicas em que a estrutura residual seja um ruído branco, isto é, que o resíduo seja uma variável aleatória independente e identicamente distribuída.

Segundo Morettin e Toloi (1989), a intervenção consiste em uma mudança de nível ou inclinação ocorrida com os dados num determinado instante do tempo, por algum motivo conhecido ou não, ou seja, por alguma interferência, a série muda de segmento em que estava se desenvolvendo num instante ou durante um período de tempo. Esses fenômenos que aparecem em algumas séries não são estimados pelo modelo ARIMA.

A intervenção, muitas vezes, pode estar obscura por três fontes de "ruídos": a tendência, sazonalidade e o erro aleatório. $\mathrm{O}$ fato de existir tendência na série pode induzir o pesquisador a tirar falsas conclusões, pois

\footnotetext{
1. Dra. em Estatística. Professora Adjunto IV. Departamento de Ciências Exatas - Universidade Federal de Lavras/UFLA - Caixa Postal 37 - $37200-000$ - Lavras, MG. safadi@ufla.br
} 
a ocorrência de uma inclinação ou uma mudança de nível na série pode ocorrer devido a uma tendência.

As primeiras propostas de se fazer uma análise de intervenção parecem ter sido feitas nas áreas de ciências sociais, com Campbell (1963) e Campbell e Stanley (1966).

Tiao et al. (1975) utilizaram a análise de intervenção para estudar dados de poluição em Los Angeles no período de janeiro de 1955 a dezembro de 1972. Os dados referiam-se a medições horárias de poluentes primários e de poluentes secundários, em diversos locais. Em 1960, houve uma possível mudança no nível de ozônio devido à abertura da autopista "Golden State Freeway", a qual pode ter afetado o tráfego no centro da cidade, e à introdução de regulamento que reduziu a proporção de hidrocarbonetos reativos na gasolina vendida em Los Angeles.

Ledolter et al., citados por Pino (1980), utilizaram a análise de intervenção para estudar dados de poluição atmosférica em New Jersey no período de janeiro de 1971 a junho de 1977 e verificaram o efeito de vários eventos, tais como leis regulamentando a emissão de CO, crise de energia em 1973 e outros.

Saboia (1976) utilizou a análise de intervenção para estudar o efeito da queda no padrão de vida sobre o índice de mortalidade infantil no município de São Paulo, e teve por objetivo verificar a influência do poder aquisitivo das famílias sobre essa série.

Pino e Morettin (1981) aplicaram a análise de intervenção para avaliar o impacto de variações climáticas e medidas de política agrícola sobre séries de produção de café no Brasil.

Bhattacharyya e Layton (1979) analisaram o efeito da introdução de legislação sobre o uso de cinto de segurança em automóveis, no Estado de Queensland (Austrália), sobre o número de mortes por acidentes rodoviários.

Borgatto e Sáfadi (2000) utilizaram o modelo de intervenção para analisar séries de transporte urbano em São Paulo, como as séries do número de assaltos e o número de acidentes nos ônibus urbanos.

Realizou-se o presente trabalho com o objetivo de estudar a quantidade da vazão de água da barragem de Furnas. Para a análise, consideraram-se modelos de Box e Jenkins (1976), sem e com o efeito de intervenção que teria ocorrido pelo aumento de chuva em determinadas épocas do ano, nas quais a quantidade de chuva foi maior do que a ocorrida em outros anos.

\section{MATERIAL E MÉTODOS}

A série proposta é a vazão $\left(\mathrm{m}^{3} / \mathrm{s}\right)$ da Barragem de Furnas - MG, coletada diariamente no período de janeiro de 1963 a dezembro de 1994. Foi realizada uma média mensal dos dados, em que cada média representa uma observação, num total de 372 .

A vazão $\left(\mathrm{m}^{3} / \mathrm{s}\right)$ está representada pelo volume de água que passa pela turbina e pelo volume de água que passa pelo vertedor.

De um modo geral, toda série temporal, $\left\{Y_{t}\right.$, $t=1: n\}$ pode ser decomposta na soma $Y_{t}=T_{t}+S_{t}+a_{t}$, em que a tendência $\left(T_{t}\right)$ pode ser entendida como um aumento ou diminuição gradual das observações ao longo de um período; a sazonalidade $\left(S_{t}\right)$ mostra flutuações ocorridas em períodos (menores que um ano), podendo ser mensal, trimestral, diária, etc. e a componente aleatória ou erro $\left(a_{t}\right)$ mostra as oscilações aleatórias irregulares. A suposição usual é que $a_{t}$ seja uma série puramente aleatória ou ruído branco independente, com média zero e variância constante.

O modelo ARIMA é um caso geral dos modelos propostos por Box e Jenkins (1976), o qual é apropriado para descrever séries não estacionárias, ou seja, séries que não possuem média constante no período de análise, nas quais os parâmetros quase sempre são pequenos. Na prática, geralmente as séries encontradas apresentam tendência e (ou) sazonalidade.

Quando a série não apresenta o componente sazonal, mas apresenta o componente tendência, ou esses componentes são homogêneos não-estacionários, ela pode ser representada por um modelo ARIMA, ou seja, o ajuste pode ser feito por um modelo sem o componente sazonal.

O modelo ARIMA considera a tendência da série temporal, tem ordem $(\mathrm{p}, \mathrm{d}, \mathrm{q})$ e pode ser representado por

$$
\phi(B)(1-B)^{d} Y_{t}=\theta(B) a_{t}
$$

sendo: $\phi(B)=1-\phi_{1} B-\phi_{2} B^{2}-\ldots-\phi_{p} B^{p} \quad$ o polinômio autorregressivo de ordem $p$; $\theta(B)=1-\theta_{1} B-\theta_{2} B^{2}-\ldots-\theta_{q} B^{q}$ o polinômio de médias móveis de ordem $q ; B$ o operador de retardo, tal que $B^{j} Y_{t}=Y_{t-j}$ e $d$ é o número de diferenças necessárias para retirar a tendência da série e transformá-la em estacionária.

Quando uma série temporal apresenta comportamento periódico em um período máximo de 12 meses, é necessário acrescentar uma componente sazonal no 
modelo. Esse intervalo periódico é chamado sazonalidade. Pode-se definir um operador diferença generalizado, quando duas observações distantes entre si de $s$ intervalos de tempo apresentam alguma semelhança, por

$$
\nabla_{s} Y_{t}=\left(1-B^{s}\right) Y_{t}=Y_{t}-Y_{t-s} .
$$

Box e Jenkins (1976) propõem o modelo sazonal multiplicativo, representado pela expressão

$\phi_{p}(B) \Phi_{P}\left(B^{s}\right)(1-B)^{d}\left(1-B^{s}\right)^{D} Y_{t}=\theta_{q}(B) \Theta_{Q}\left(B^{s}\right) a_{t}$,

denotado por SARIMA(p,d,q)x(P,D,Q), em que $\Phi_{P}(B)$ e $\Theta_{\mathrm{Q}}(B)$ são os polinômios sazonais autorregressivos e de médias móveis de ordens $P$ e $Q$, respectivamente, e $D$ é o número de diferenças de "lag" $s$ necessárias para retirar a sazonalidade da série.

O termo intervenção foi introduzido por Glass (1972), baseado em Box e Tiao (1965), que já utilizava esses métodos, mas não com o termo intervenção.

Segundo Jenkins (1979), " os métodos de intervenção representam generalizações de métodos usados para análise de dados, usualmente não expressos na forma de séries temporais, aos quais os estatísticos referem-se pelo título geral de Delineamento e Análise de Experimentos." Apesar de o modelo parecer simples, ele descreve um grande número de efeitos simultaneamente.

O modelo proposto para a análise de intervenção é calculado pela expressão

$$
Y_{t}=\sum_{i=1}^{K} \mathrm{v}_{i}(B) x_{i, t}+n_{t}
$$

em que $Y_{t}$ é a variável-resposta do modelo; $k$ é o número de intervenções da série; $v_{i}(B)$ é o valor da função de transferência; $x_{i, t}$ é a variável binária e $n_{t}$ é o ruído do modelo, representado por um modelo ARIMA.

\section{RESULTADOS E DISCUSSÃO}

Na Figura 1 representa-se a vazão mensal da Barragem de Furnas, e com uma análise visual não é possível afirmar que exista sazonalidade, mas sabe-se que essa, se existir, pode ser de 12 meses devido ao período de chuvas; quanto à existência da tendência, também não é possível fazer nenhuma afirmação, sendo necessária a aplicação do teste de Cox-Stuart (MORETTIN e TOLOI, 1985).

Pelo teste de Cox-Stuart, observou-se a presença da componente tendência. Assim, para tornar a série estacionária, fez-se a primeira diferença nos dados. Na Figura 2 apresentam-se a série de vazões diferenciada e a função de autocorrelação correspondente.

$\mathrm{Na}$ Figura 2 é possível observar que embora a série não apresente sazonalidade, existe correlação significativa nos "lags" múltiplos de 12 , o que levou à utilização do modelo SARIMA.

Pelos gráficos da função de autocorrelação e autocorrelação parcial da série diferenciada (Figura 3), ajustouse um modelo SARIMA $(1,1,1) \times(2,0,0)_{12}$, ou seja, o modelo $\left(1-\phi_{1} B\right)\left(1-\Phi_{1} B^{12}-\Phi_{2} B^{24}\right)(1-B) Y_{t}=\left(1-\theta_{1} B\right) a_{t}$, em que $a_{t}$ é o resíduo que é independente e identicamente distribuído, portanto, um ruído branco, conforme Figura 4.

$\mathrm{Na}$ Tabela 1 verificam-se os valores dos coeficientes do modelo e seus respectivos desviospadrão.

TABELA 1 - Coeficientes do modelo SARIMA $(1,1,1) \times(2,0,0)_{12}$ e respectivos desvios-padrão.

\begin{tabular}{ccc}
\hline Coeficientes & Estimativa & Desvio-Padrão \\
\hline$\phi_{1}$ & 0,62122 & 0,06322 \\
\hline$\theta_{1}$ & 0,96199 & 0,03201 \\
\hline$\Phi_{1}$ & 0,21227 & 0,05422 \\
\hline$\Phi_{2}$ & 0,10663 & 0,0527 \\
\hline
\end{tabular}

Para o ajuste do modelo com intervenção, observaram-se novamente as Figura 1 e 2, notando que os acréscimos encontrados nas observações 197 (maio de 1980), 233 (maio de 1983) e 330 (junho de 1991) podem ser consideradas como possíveis intervenções, já que esses aumentos não desapareceram na série sem tendência.

Assim, ajustou-se o modelo SARIMA $(1,1,1) \times(1,0,0)_{12}$ com 3 intervenções , $\mathrm{w}_{1}, \mathrm{w}_{2}$ e $\mathrm{w}_{3}$, correspondendo respectivamente às observações 197, $233 \mathrm{e}$ 330. Na Tabela 2 observam-se os valores estimados para os coeficientes, assim como os respectivos errospadrão. 


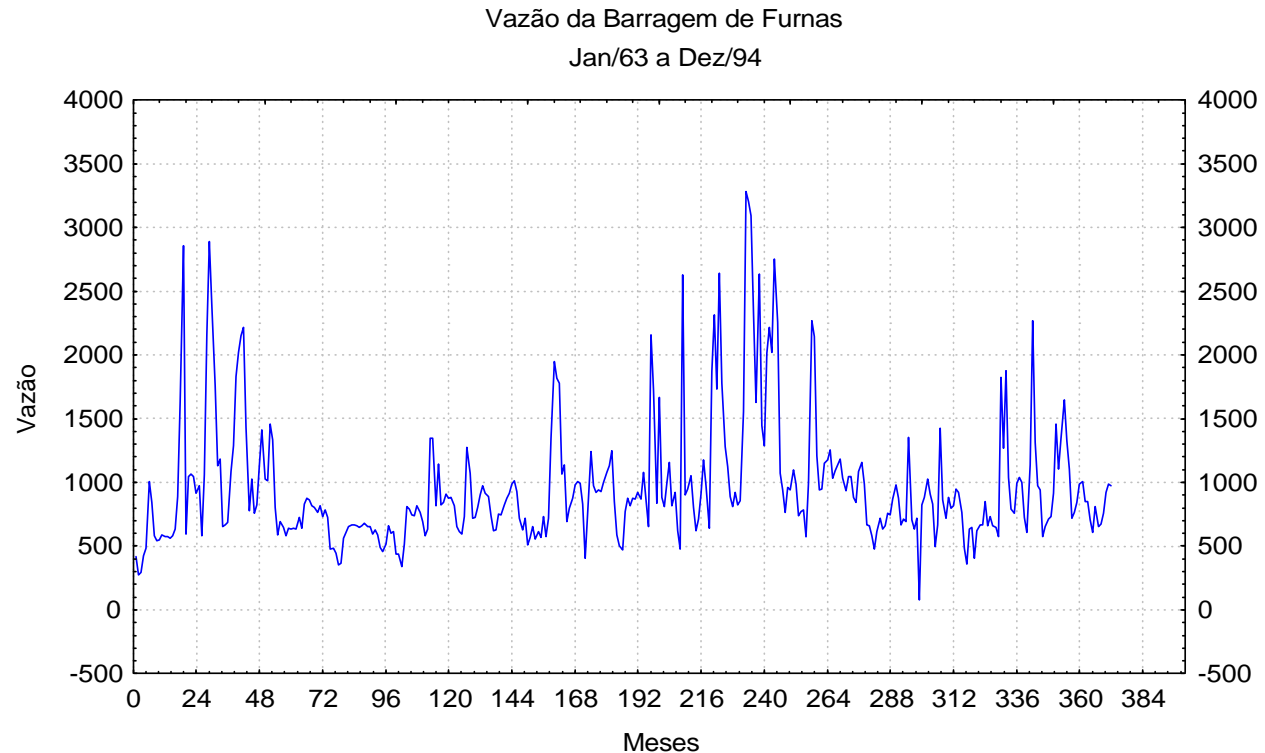

FIGURA 1 - Gráfico da série de vazão de água na Barragem de Furnas: período de jan./ 1963 a dez./ 1994.
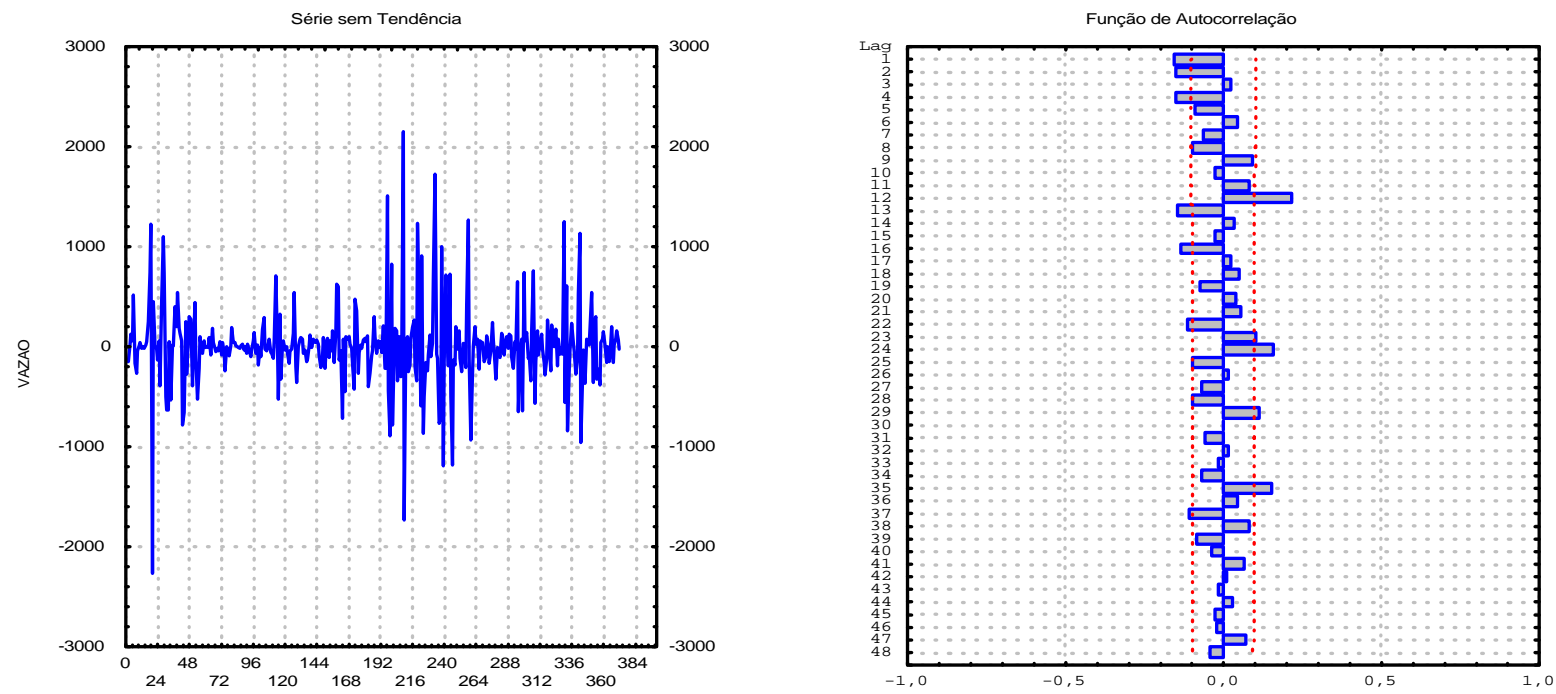

FIGURA 2 - Gráfico da série sem tendência e da respectiva função de autocorrelação. 

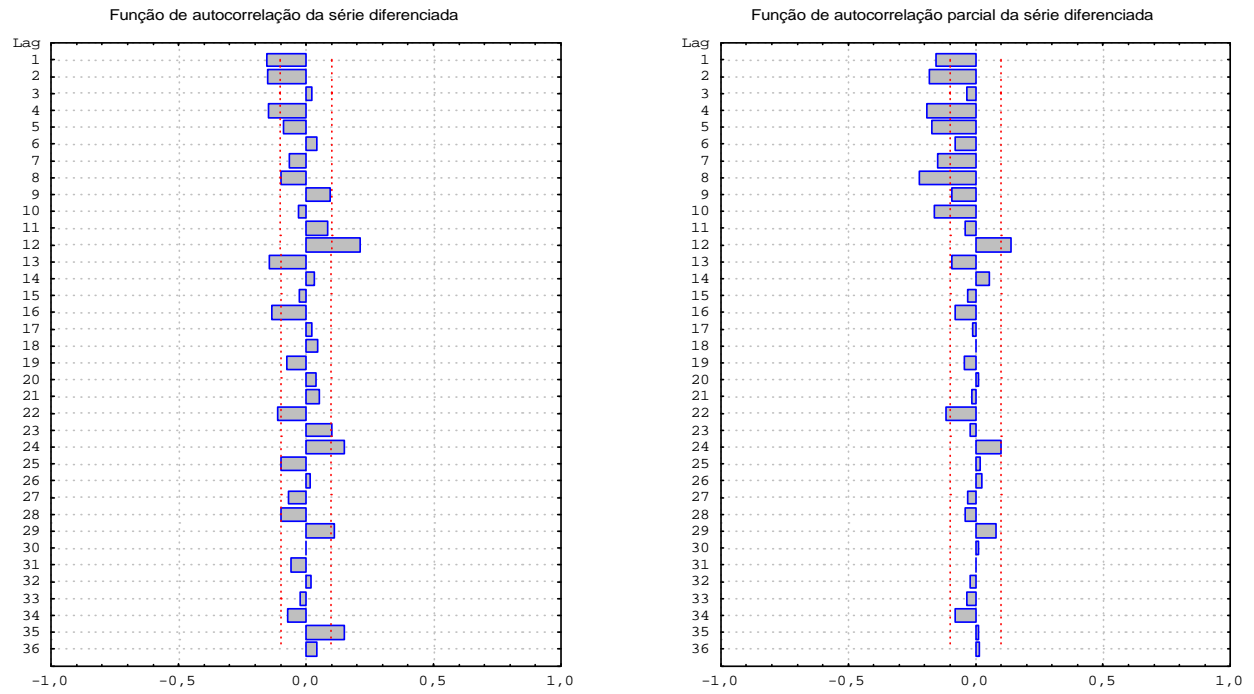

FIGURA 3 - Função de autocorrelação e autocorrelação parcial da série diferenciada.

Função de autocorrelação do resíduo

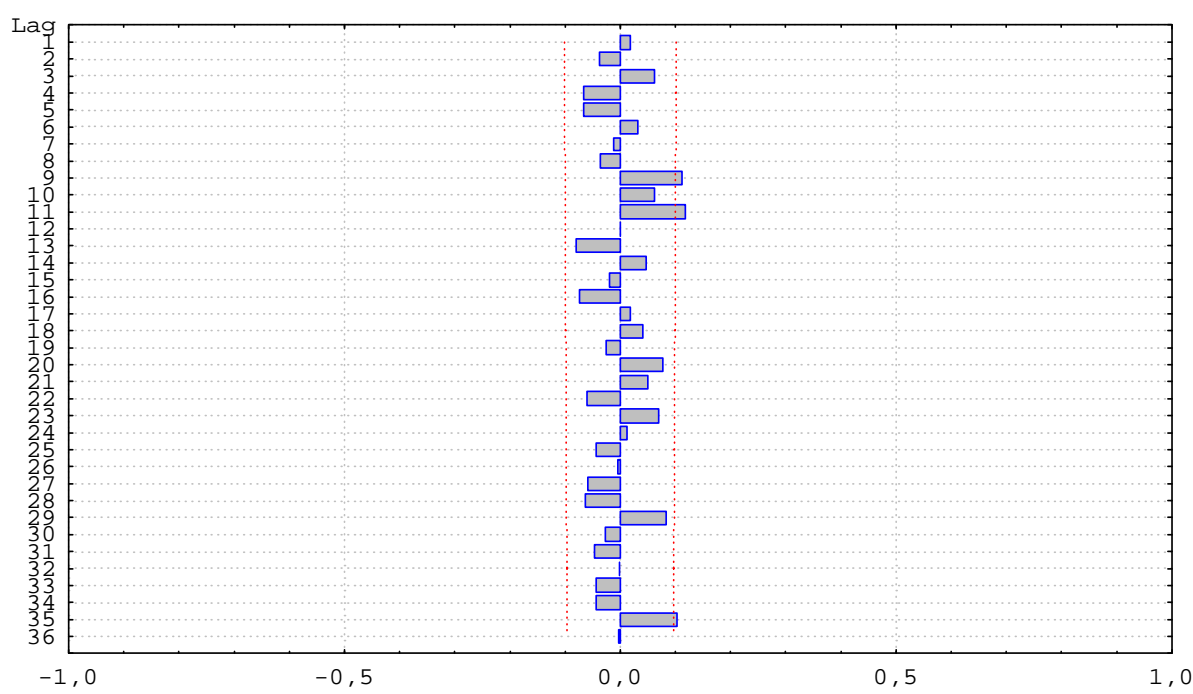

FIGURA 4 - Função de autocorrelação do resíduo (ruído branco). 
TABELA 2 - Estimativas para os coeficientes do modelo SARIMA(1,1,1)x(1,0,0) ${ }_{12}$ com intervenções em maio/80, junho/81 e abril/91 e respectivos desvios-padrão.

\begin{tabular}{ccc}
\hline Coeficiente & Estimativa & Desvio-Padrão \\
\hline$\phi_{1}$ & 0,54504 & 0,05865 \\
\hline$\theta_{1}$ & 0,90393 & 0,03062 \\
\hline$\Phi_{1}$ & 0,22825 & 0,05299 \\
\hline $\mathrm{w}_{1}$ & 626,650 & 280,650 \\
\hline $\mathrm{w}_{2}$ & 1295,60 & 302,000 \\
\hline $\mathrm{w}_{3}$ & 665,240 & 275,760 \\
\hline
\end{tabular}

O modelo de intervenção com seus parâmetros estimados pode ser escrito como:

$$
Y_{t}=626,65 x_{1, t}+1295,6 x_{2, t}+665,24 x_{3, t}+\frac{(1-0,90393 B)}{(1-B)\left(1-0,22825 B^{12}\right)(1-0,54504 B)} a_{t}
$$

e para as variáveis "dummy" $x_{1, t}, x_{2, \mathrm{t}}$ e $x_{3, \mathrm{t}}$ tem-se

$$
x_{1, t}=\left\{\begin{array}{l}
1, \text { se } t=197 \\
0, \text { c.c }
\end{array}, \quad x_{2, t}=\left\{\begin{array}{l}
1, \text { se } t=233 \\
0, \text { c.c }
\end{array} \text { e } x_{3, t}=\left\{\begin{array}{l}
1, \text { se } t=330 \\
0, \text { c.c }
\end{array}\right. \text {. }\right.\right.
$$

Pode-se dizer que houve um aumento médio na vazão da Barragem de Furnas de $626 \mathrm{~m}^{3} / \mathrm{s}$ em maio de 1980 , de $1296 \mathrm{~m}^{3} / \mathrm{s}$ em maio de 1983 e de $665 \mathrm{~m}^{3} / \mathrm{s}$ em junho de 1991.

\section{CONCLUSÕES}

Os modelos de séries temporais podem ser úteis para descrever a série de vazão de água da Barragem de Furnas.

Observa-se a existência de um componente sazonal de multiplicidade 12 em relação à quantidade de chuva que ocorre nos meses de cada ano, sendo a época de maior quantidade de chuva no meio do ano (junho e julho), levando ao ajuste de modelo SARIMA.

Entre os modelos ajustados com e sem intervenção, observou-se que os modelos com intervenção fornecem informações adicionais, ou seja, nos anos de 1980, 1983 e 1991 houve uma vazão maior do que a esperada nos meses de maio e junho.

\section{REFERÊNCIAS BIBLIOGRÁFICAS}

BHATTACHARYYA, M. N.; LAYTON, A. P. Effectiveness of seat belt legislation on the queensland road toll: an Australian case study in intervention analysis. Journal of the American Statistical Association, Alexandria, v. 74, n. 367, p. 596-603, Sept. 1979.

BORGATTO, A. F.; SÁFADI, T. Análise de intervenção em séries temporais: aplicações em transporte urbano. Revista Brasileira de Estatística, Rio de Janeiro, v. 61, n. 215, p. 81-102, 2000 .

BOX, G. P.; JENKINS, G. M. Time series analysis, forecasting and control. San Francisco: Holden-Day, 1976.

BOX, G. P.; TIAO, G. C. A change in level of non stationary time-series. Biometrika, London, v. 52, n. 1/2, p. 181-192, 1965. 
CAMPBELL, D. T. From description to experimentation: interpreting trends as quasi-experiments. In: HARRIS, C. W. (Ed.). Problems of measuring change: proceedings. Madison: University of Wisconsin, $1963.259 \mathrm{p}$.

CAMPBELL, D. T.; STANLEY, J. C. Experimental and quasi-experimental designs for research. Dallas: H. Mifflin, 1966. 84 p

GLASS, G. V. Estimating the effects of intervention into a non-stationary time series. American Educational Research Journal, Washington, v. 9, n. 3, p. 463-477, 1972.

JENKINS, G. M. Pratical experiences with modeling and forecasting time series. Jerrey: Channel Island, 1979.

MORETTIN, P. A.; TOLOI, C. M. C. Previsão de séries temporais. São Paulo: Atual, 1985.
MORETTIN, P. A.; TOLOI, C. M. C. Modelos de função de transferência. São Paulo: ABE, 1989.

PINO, F. A. Análise de intervenção em séries temporais: aplicações em economia. São Paulo: IME-USP, 1980.

PINO, F. A.; MORETTIN, P. A. Intervention analysis applied to brazilian milk and coffee time series. São Paulo: IME-USP, 1981.

SABOIA, J. L. M. Mortalidade infantil e salário mínimo: uma análise de intervenção para o município de São Paulo. Revista de Administração de Empresas, Rio de Janeiro, v. 16, n. 3, p. 47-50, maio/jun. 1976.

TIAO, G. C.; BOX, G. E. P.; HAMMING, W. J. Analysis of Los Angeles photochemical smong data: a statistical overview. Journal of the Air Polution Control Association, [S.1.], v. 25, n. 3, p. 260-268, Mar. 1975. 